\title{
氨基功能化整体材料在磷酸化肽快速可控 富集中的应用
}

\author{
徐林楠 ${ }^{1,2}$, 白玉 ${ }^{1 *}$, 刘虎威 $1^{*}$ \\ 1. 北京大学化学与分子工程学院分析化学研究所, 生物有机与分子工程教育部重点实验室, 北京分子科学国家实验室, 北京 100871 ; \\ 2. 北京航天试验技术研究所, 北京 100074 \\ *联系人, E-mail: yu.bai@pku.edu.cn; hwliu@pku.edu.cn \\ 收稿日期: 2017-07-23; 接受日期: 2017-09-21; 网络版发表日期: 2017-12-26 \\ 国家自然科学基金(批准号: 21575007, 21527809,21322505)资助
}

\begin{abstract}
摘要针对质谱在磷酸化肽分析过程中检测灵敏度低的问题, 本文在移液器吸头尖端原位制备了氨基功能 化整体材料,并将其应用于磷酸化肽的快速富集. 基于该材料所建立的磷酸化肽富集方法操作简便、分析时间 短, 并且能够有效避免富集过程中材料与溶液分离不完全所导致的样品损失等问题. 而在不同组成的上样溶液 条件下,氨基功能化整体材料对单磷酸化肽和多磷酸化肽表现出了不同的磷酸化肽富集选择性, 因而借助于对 上样溶液组成的调控,该材料可以分别实现对磷酸化肽的全富集以及对多磷酸化肽的选择性富集.
\end{abstract}

关键词氨基功能化整体材料, 磷酸化肽, 快速富集,可控富集选择性

作为存在最为普遍的一类蛋白质翻译后修饰, 磷 酸化在蛋白质活性和功能的调控中扮演着重要的角 色, 并参与到细胞生长、分裂、分化、调亡等几乎所 有的细胞生命活动中 ${ }^{[1,2]}$. 而蛋白质磷酸化的异常, 也 与多种疾病的发生和发展存在着密切的联系 ${ }^{[3,4]}$. 建立 有效的蛋白质磷酸化分析手段, 对于深入理解蛋白质 磷酸化及其相关生物学意义具有十分重要的价值. 在 磷酸化蛋白质组学相关的研究中, 质谱由于其分析灵 敏度、分析速度以及结构鉴定能力的优势而具有强 大的应用潜力 ${ }^{[5,6]}$. 然而, 由于天然丰度低 ${ }^{[7]}$ 、离子化 能力差 ${ }^{[8]}$ 以及高丰度非磷酸化肽所带来的干扰 ${ }^{[9]}$ 等因 素限制了质谱对磷酸化肽的直接分析能力, 借助样品 前处理过程实现磷酸化肽的富集, 从而提高其质谱分
析灵敏度已成为磷酸化肽质谱分析的必要前提.

对样品中的低丰度磷酸化肽进行富集, 既能够提 高用于质谱分析的磷酸化肽样品浓度, 又可以有效去 除各种干扰物质的影响. 对磷酸化肽富集材料的开发, 则是实现对磷酸化肽特异、高效富集的关键问题. 近 年来, 抗体/结构域修饰材料 ${ }^{[10,11]}$ 、印迹材料 ${ }^{[12]}$ 、离子 交换材料 ${ }^{[13]}$ 、固定金属离子材料 ${ }^{[14]}$ 、金属氧化物材 料 ${ }^{[15]}$ 、金属有机框架材料 ${ }^{[16]}$ 等多种基于不同类型富 集亲和作用的富集材料先后被开发出来并应用于磷 酸化肽富集中. 不同材料与磷酸基团之间亲和作用强 弱存在一定的区别, 导致不同材料具有各异的对单磷 酸化肽和多磷酸化肽的富集选择性 ${ }^{[17]}$. 具有较强亲和 作用的富集材料可以同时富集单磷酸化肽和多磷酸

引用格式: 徐林楠, 白玉, 刘虎威. 氨基功能化整体材料在磷酸化肽快速可控富集中的应用. 中国科学: 生命科学, 2018, 48: 207-214

Xu L N, Bai Y, Liu H W. Amino-functionalized monolithic tips for rapid phosphopeptide enrichment with tunable selectivity (in Chinese). Sci Sin Vitae, 2018, 48: 207-214, doi: 10.1360/N052017-00171 
化肽, 从而实现较为全面的磷酸化肽分析. 而亲和作 用较弱的材料, 能够选择性富集具有较多磷酸化位点 的多磷酸化肽, 降低待分析样品中磷酸化肽组成的复 杂度, 增强质谱对含量更少、离子化能力更差的多磷 酸化肽的检出能力和分析效果 ${ }^{[18]}$, 从而对磷酸化肽的 全富集分析实现良好的补充. 然而, 由于以金属氧化 物和固定金属离子材料为代表的磷酸化肽富集材料 多具有固定的磷酸化肽富集选择性, 两种富集方式的 同时应用通常需要借助于不同材料的组合才能够完 成 ${ }^{[19,20]}$. 在本课题组之前的工作中, 报道了胍基功能化 石墨烯 ${ }^{[21]}$ 以及酰肼基功能化单分散硅胶微球 ${ }^{[2]}$ 等两 种具有可控富集选择性的磷酸化肽富集材料, 并通过 对富集过程中上样溶液条件的控制, 实现了利用单一 材料对全部磷酸化肽的同时富集以及对多磷酸化肽 的选择性富集.

在磷酸化肽富集材料的应用形式上, 最常用的方 式是将材料颗粒分散于样品溶液中, 并通过一段时间 的混合孵育, 使其充分吸附样品溶液中的磷酸化肽, 富 集完成后, 借助于离心或外加磁场的方式将材料与溶 液相分离. 由于富集过程中上样、洗涤、洗脱等多个 操作步骤的存在, 富集材料需要在溶液中进行多次分 散和聚集的循环, 良好的材料分散性尽管可以保证材 料能够与溶液充分接触, 但也为材料与溶液的分离带 来困难, 增加分离操作所需的时间. 富集材料与溶液 分离的不完全, 更会造成样品损失、溶液成分变化、 残留材料再吸附等问题的发生, 从而严重影响分析结 果的准确性. 将材料颗粒制成填充柱的形式有助于解 决上述问题, 例如, 现有商品化的Ziptip ${ }^{\circledR}$ 等, 便是将反 相吸附材料或离子交换吸附材料填充于移液器洗头 中, 以实现对萃取富集操作的简化. 而针对于磷酸化 肽的特异性富集, 则有报道通过吸头尖端填充金属氧 化物材料或固定金属离子材料颗粒等来实现 ${ }^{[23,24]}$. 然 而将颗粒材料制成吸头填充柱的方式仍存在着材料 填充不均匀、固定方式复杂以及固定不充分等问题. 整体材料具有材料基底与孔道结构双连续的特点, 其 连续的基底结构使其易于以填充柱的形式应用于样 品前处理中, 同时减少了操作过程中发生材料流失的 可能性; 而其连续的孔道结构既为其提供了较大的比 表面积以及吸附容量, 又可以减少溶液通过所需的压 力, 使操作条件得到简化. 这些特点使得整体材料在 包括萃取富集在内的样品分离领域有着广阔的应用
前景 ${ }^{[25,26]}$.

本工作将整体材料在快速分离分析中的优势与可 控选择性的材料对磷酸化肽富集能力相结合, 设计并 制备了原位生长于移液器吸头尖端的氨基功能化整体 材料, 并将其应用于对磷酸化肽的快速、双功能富集. 相比于将材料颗粒分散于溶液中的操作方式, 将其填 充于移液器吸头尖端简化了富集操作, 并有效缩短了 富集操作所需的时间. 与此同时,氨基功能化整体材 料在不同的上样溶液条件下还表现出了不同的磷酸 化肽富集选择性, 通过对上样溶液组成的选择, 调控 材料对单磷酸化肽和多磷酸化肽的富集选择性, 从而 实现对全部磷酸化肽以及多磷酸化肽的双功能富集.

\section{1 材料与方法}

\section{1 试剂与仪器}

二甲基丙烯酸乙二醇酯(纯度 $98 \%$, 含 $0.1 \mathrm{mg} / \mathrm{g}$ 稳定 剂4-甲氧基苯酚)及甲基丙烯酸(纯度 $>99 \%$,含 $0.1 \mathrm{mg} / \mathrm{g}$ 稳定剂4-甲氧基苯酚)购自Alfa Aesar (美国). 2-氨基 乙基甲基丙烯酸酯盐酸盐(纯度 $90 \%$, 含 $0.5 \mathrm{mg} / \mathrm{g}$ 稳定 剂吩噻嗪)和 2,5 -二羟基苯甲酸(纯度 $99 \%$ )购自北京百 灵威科技有限公司. 乙酸(分析纯)、磷酸(优级纯)及 1-十二醇(化学纯)购自北京化工厂.甲酸(分析纯)及 偶氮二异丁腈(化学纯)购自国药集团化学试剂有限公 司. 甲苯(分析纯)购自北京市通广精细化工公司. 胰 蛋白酶(TPCK处理)、 $\alpha$-酪蛋白(纯度 $\geq 70 \%) 、 \beta$-酪蛋 白(纯度 $>98 \%$ )、乙腈(HPLC级)及甲醇(HPLC级)购自 Sigma-Aldrich (美国). 碳酸氢铵(纯度 $99.5 \%$ )购自Fluka (瑞士). 三氟乙酸(99\%)购自Acros Organics (美国). 脱 脂牛奶购自内蒙古伊利实业集团股份有限公司. 纯净 水购自杭州娃哈哈集团有限公司.

所制备材料中碳、氢、氮元素的质量分数利用元 素分析仪(vario EL, Elementar Analysensysteme GmbH, 德国)测定. 红外吸收光谱利用红外光谱仪(Tensor 27 FT-IR, Bruker, 德国)通过将研磨后的材料与干燥澳化 钾混合压片测定. 比表面积通过比表面孔分布测定仪 (ASAP2020, Micromeritics, 美国)测定 $p / p_{0}$ 在0.05 0.30 的吸附等温线并通过BET方程拟合进行计算, 测试前 样品在真空条件 $70^{\circ} \mathrm{C}$ 活化以除去表面吸附的气体分 子. 所富集样品使用基质辅助激光解吸附/离子化-飞 
行时间质谱(ultraflex MALDI-TOF/TOF MS, Bruker)分 析其组成.

\section{2 材料合成}

制备氨基功能化整体材料时, 首先在 $50^{\circ} \mathrm{C}$ 加热条 件下将 $23.8 \mathrm{mg}$ 2-氨基乙基甲基丙烯酸酯盐酸盐溶解

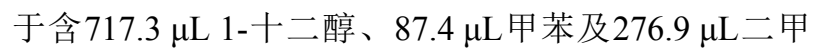
基丙烯酸乙二醇酯的混合溶液中, 并超声和通氮气各 $10 \mathrm{~min}$ 以除尽中的溶液中溶解的氧气. 之后加入 $4 \mathrm{mg}$ 偶氮二异丁腈, 超声使其溶解, 从而得到用于后续聚 合反应的混合反应液.

氨基功能化材料在移液器吸头内的原位生长 按图 1所示的流程进行: 首先在 $2 \mathrm{~mL}$ 离心管的顶盖 钻一直径约为 $5 \mathrm{~mm}$ 的孔, 使容量为 $200 \mu \mathrm{L}$ 的移液器吸 头刚好能够嵌入至后端突出部分. 同时, 在 $2 \mathrm{~mL}$ 离心 管侧壁另钻一小孔, 以保证管内外压力的平衡. 向 $2 \mathrm{~mL}$ 离心管中加入 $250 \mu \mathrm{L}$ 上述混合反应液, 然后在其顶盖 的孔中嵌入 $200 \mu \mathrm{L}$ 移液器吸头, 移液器洗头尖端刚好 接触反应液表面. 约 $5 \mu \mathrm{L}$ 反应液可以在毛细作用下进 入并停留于移液器吸头尖端. 之后将这一组合反应装 置置于 $10 \mathrm{~mL}$ 离心管中, 封闭以隔绝外界空气, 然后置 于 $70^{\circ} \mathrm{C}$ 烘箱内反应 $24 \mathrm{~h}$, 使离心管内与移液器吸头尖 端的反应液一并发生聚合反应. 反应完成后取出移液 器吸头, 离心作用下使甲醇和乙腈多次流过吸头尖端 的整体材料, 以洗去材料中包含的未发生聚合反应的 溶剂和致孔剂等. 离心管内聚合的整体材料取出并研 磨后, 使用甲醇和乙腈多次洗涤, 并于 $40^{\circ} \mathrm{C}$ 过夜烘干.

\section{3 样品制备}

胰蛋白酶及标准磷酸化蛋白 $\beta$-酪蛋白酶解液分 别以 $1 \mathrm{mg} / \mathrm{mL}$ 的浓度溶于 $50 \mathrm{mmol} / \mathrm{L}$ 碳酸氢铵溶液中. 向 $1 \mathrm{~mL} \beta$-酪蛋白溶液中加入 $25 \mu \mathrm{L}$ 胰蛋白酶溶液, 于 $37^{\circ} \mathrm{C}$ 酶解 $16 \mathrm{~h}$, 得到标准磷酸化蛋白 $\beta$-酪蛋白.

$30 \mu \mathrm{L}$ 脱脂牛奶使用 $970 \mu \mathrm{L} 50 \mathrm{mmol} / \mathrm{L}$ 碳酸氢铵溶 液稀释, 之后在 $20000 \times g$ 下离心 $25 \mathrm{~min}$. 取上清液于 $100^{\circ} \mathrm{C}$ 下变性 $5 \mathrm{~min}$, 冷却后加入 $30 \mu \mathrm{L}$ 胰蛋白酶溶液. 于 $37^{\circ} \mathrm{C}$ 酶解反应 $16 \mathrm{~h}$, 得到脱脂牛奶酶解液样品.

\section{4 磷酸化肽富集}

在进行磷酸化肽富集操作时, 将尖端生长有氨基
功能化整体材料的移液器吸头嵌入至顶盖打孔的离心 管顶盖上, 以便完成富集过程中的离心操作. 富集前, 首先分别使用乙腈以及与稀释酶解液所用的相同的上 样溶液对材料进行活化和平衡. 蛋白酶解液使用一定 组成的上样溶液稀释至特定的浓度, 取 $200 \mu \mathrm{L}$ 加入至 吸头内, 在离心作用下使其以约 $30 \mu \mathrm{L} / \mathrm{min}$ 的流速通过 吸头尖端的整体材料, 从而完成对稀释酶解液中磷酸 化肽的吸附. 之后使用相同组成的上样溶液洗涤材料 2 次, 以除去材料表面的非特异性吸附. 最后, 使用 $5 \mu \mathrm{L}$ 组成为 $20 \mathrm{mg} / \mathrm{mL} 2,5$-二羟基苯甲酸、 $50 \%$ 乙腈及 $1 \%$ 磷 酸的MALDI质谱基质溶液对材料表面吸附的磷酸化肽 进行洗脱. 取 $1 \mu \mathrm{L}$ 收集得到的洗脱液, 滴于MALDI-MS 的靶板上, 待其干燥后使用MALDI-ToF MS进行分析. 而洗脱完成后的材料, 则可以利用乙腈/水 $1: 1,5 \%$ 三氟 乙酸溶液进行再生, 以便于多次重复利用.

\section{2 结果与讨论}

\section{1 材料合成与表征}

在制备氨基功能化整体材料时, 使用含不饱和键 的甲基丙烯酸类衍生物为聚合反应的单体, 利用加热 条件下偶氮二异丁腈分解产生的自由基引发自由基链 式聚合反应. 其中, 2-氨基乙基甲基丙烯酸酯用于在材 料中引入氨基, 而二甲基丙烯酸乙二醇酯则用于单体 之间的相互交联. 由于氧气会猝灭链式聚合反应中的 自由基, 而发生于移液器吸头内的反应体系较小使聚 合反应更容易为空气中的氧气所猝灭, 因而在移液器 吸头内制备整体材料时, 借助于两个离心管组成的套 管结构, 来隔绝外界空气并扩大反应体系以消除氧气 对聚合反应的影响. 借助该方法, 制备了如图1所示的 原位生长于移液器吸头尖端的氨基功能化整体材料. 材料的红外吸收光谱如图2所示, 吸收谱图中

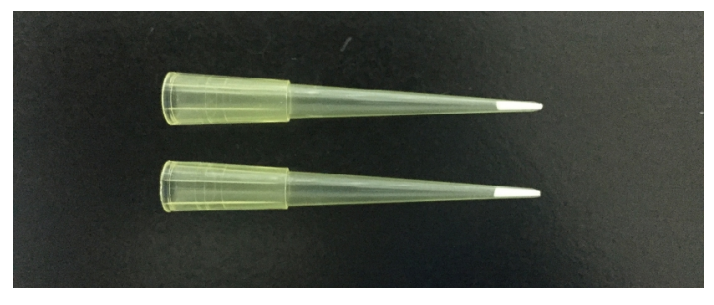

图 1 原位生长于移液器吸头尖端的氨基功能化整体材 料(网络版彩图) 


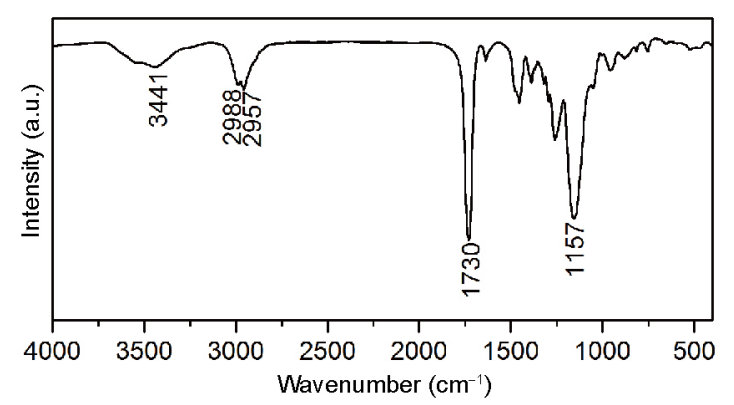

图 2 氨基功能化整体材料的红外吸收光谱图

$\sim 3400 \mathrm{~cm}^{-1}$ 附近的吸收峰为氨基 $\mathrm{N}-\mathrm{H}$ 键的伸缩振动峰; $\sim 2950 \mathrm{~cm}^{-1}$ 附近的吸收峰为聚合物骨架上烷基 $\mathrm{C}-\mathrm{H}$ 键 以及质子化氨基 $\mathrm{N}-\mathrm{H}$ 键伸缩振动的吸收峰; $1730 \mathrm{~cm}^{-1}$ 处的强吸收峰为酯键中 $\mathrm{C}=\mathrm{O}$ 伸缩振动吸收; $1150 \mathrm{~cm}^{-1}$ 处吸收峰为 $\mathrm{C}-\mathrm{O}, \mathrm{C}-\mathrm{N}$ 键的伸缩振动吸收峰. 上述结 果与目标产物氨基功能化整体材料的结构完全相符. 元素分析结果表明, 材料中的 $\mathrm{C}, \mathrm{H}, \mathrm{N}$ 元素质量分数 分别为 $58.66 \%, 7.30 \%$ 和 $0.74 \%$. 由于红外吸收光谱中 $2200 \mathrm{~cm}^{-1}$ 处观察不到氰基的特征强吸收峰, 由此可知 $\mathrm{N}$ 元素基本均来自于材料中修饰的氨基, 据此可以计 算出材料中氨基的密度为 $0.53 \mathrm{mmol} / \mathrm{g}$. 通过测定氮气 吸附等温曲线计算出材料的BET比表面积为 $65 \mathrm{~m}^{2} / \mathrm{g}$.

\section{2 快速、双功能磷酸化肽富集}

利用氨基功能化整体材料进行磷酸化肽富集的 操作流程如图3所示. 样品溶液、洗涤溶剂、洗脱溶 剂等依次加入吸头内并使其在低速离心下以可控流 速流过整体材料柱床的方式, 实现材料与上述溶液的 充分接触, 以完成材料对磷酸化肽的吸附、对材料非 特异性吸附的洗涤以及对磷酸化肽的洗脱. 该富集方 式使得整个富集操作流程仅需 $20 \mathrm{~min}$ 即可完成, 便于 实现磷酸化肽的快速富集分析, 也避免了材料与溶液 分离不完全导致的样品损失.

首先使用标准磷酸化蛋白 $\beta$-酪蛋白的酶解液试验 材料对磷酸化肽的富集效果. 富集前 $\beta$-酪蛋白酶解液 直接质谱分析结果显示, 谱图中存在大量非磷酸化肽 的质谱信号, 检出的磷酸化肽数目及信号强度均十分 有限, 无法实现对磷酸化肽的有效检出和分析(图4A). 利用氨基功能化整体材料对酶解液中的磷酸化肽进 行富集时, 通常需要加入一定浓度的小分子有机酸来 调节溶液酸度, 以减少多肽分子上羧基所造成的非特

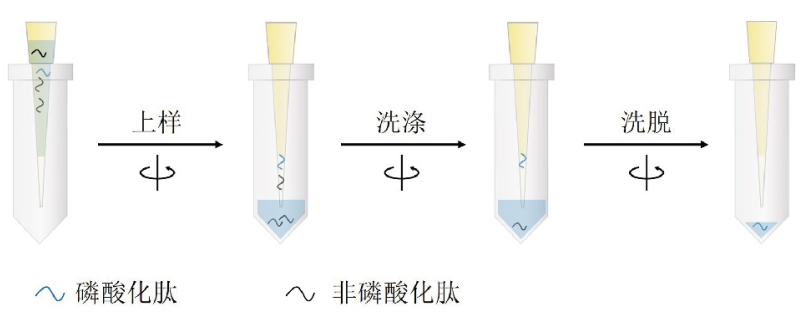

图 3 氨基功能化整体材料富集磷酸化肽流程示意图 (网络版彩图)

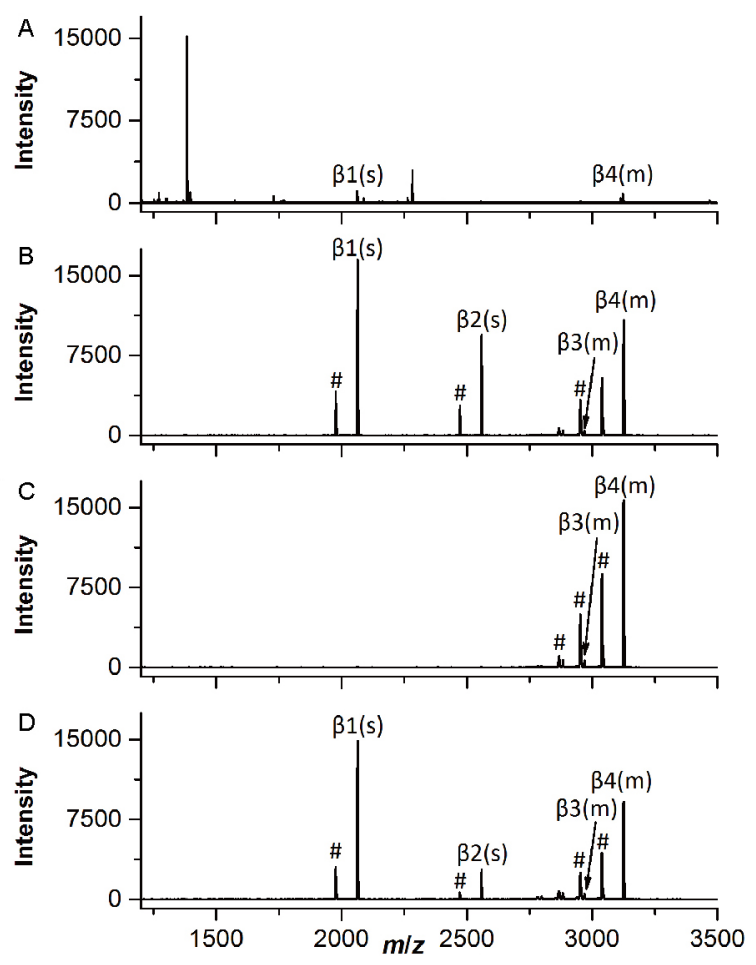

图 4 氨基功能化整体材料富集 $\beta$-酪蛋白酶解液 $\left(4 \times 10^{-7} \mathrm{~mol} / \mathrm{L}\right)$ 中磷酸化肽的质谱图

A: $\beta$-酪蛋白酶解液富集前的质谱图; B: 上样溶液组成为 $60 \%$ 乙腈、 $0.05 \mathrm{~mol} / \mathrm{L}$ 乙酸下的富集结果; $\mathrm{C}$ : 上样溶液组成为 $60 \%$ 乙腈、 $1 \%$ 甲 酸下的富集结果; D: 材料重复利用 8 次后在上样溶液组成为 $60 \%$ 乙 腈、 $0.05 \mathrm{~mol} / \mathrm{L}$ 乙酸下的富集结果

异性吸附. 通过尝试不同种类的有机酸添加剂并对其 浓度进行优化, 发现氨基功能化整体材料在两种不同 的溶剂条件下表现出了截然不同的磷酸化肽富集选 择性. 在组成为 $60 \%$ 乙腈、 $0.6 \%$ 乙酸的溶液条件下, 材 料可以同时富集到酶解液中的两条单磷酸化肽和两 条多磷酸化肽, 谱图中没有非磷酸化肽的信号(图4B); 当溶液中添加的有机酸替换为 $1 \%$ 甲酸时, 材料选择性 
地富集到两条多磷酸化肽, 而酶解液中的非磷酸化肽 和单磷酸化肽则均不会为材料所吸附 (图 4C). 这一结 果表明, 该材料具有随上样溶液条件而变化的磷酸化 肽富集选择性, 可在不同的上样溶液条件下分别实现 对磷酸化肽的全富集以及对多磷酸化肽的选择性富 集, 因而具有双功能磷酸化肽富集能力.

材料可变的富集选择性, 来自于不同上样溶液酸 度条件下磷酸化肽上磷酸基团解离程度以及材料对 磷酸化肽吸附作用强弱的差异. 材料对磷酸化肽的富 集能力, 来自于材料表面质子化的氨基与磷酸化肽上 解离磷酸基之间的静电吸引力. 在乙酸溶液条件下, 溶液酸度较弱, 磷酸根解离较为完全, 质子化氨基与 单个磷酸基之间的静电吸引足以保证材料对磷酸化 肽的吸附, 此时材料可以同时富集单磷酸化肽和多磷 酸化肽; 而在甲酸溶液条件下, 磷酸基的解离由于溶 液酸度的提高而受到抑制, 从而削弱了材料对磷酸化 肽的吸附能力, 因而此时仅有包含多个磷酸基团的多 磷酸化肽能够被材料所富集.

在添加浓度更高、酸性更强的小分子有机酸的 上样溶液条件下, 材料表面吸附的所有磷酸化肽由于 磷酸基团的完全质子化而均会解吸附. 基于此, 在富 集完成后, 使用含 $50 \%$ 乙腈、 $5 \%$ 三氟乙酸的溶液对材 料进行洗涤, 可以实现材料的再生和重复利用. 所制 备的氨基功能化整体材料在不同的溶液条件下经过 8 次富集和再生的循环后, 仍然具有完好的磷酸化肽富 集能力, 在乙酸溶液条件下仍然能够同时实现对 4 条 单磷酸化肽和多磷酸化肽的有效富集(图4D).

对材料的富集检出限的考察, 在添加乙酸的溶液 条件下, 当 $\beta$-酪蛋白酶解液的初始浓度为 $5 \times 10^{-10} \mathrm{~mol} / \mathrm{L}$ 时, 经材料富集后可同时检出一条单磷酸化肽和一条 多磷酸化肽; 而当 $\beta$-酪蛋白酶解液的初始浓度进一步 降低至 $2 \times 10^{-10} \mathrm{~mol} / \mathrm{L}$ 时, 在富集后的样品中仍可检出 其中的单磷酸化肽. 而在添加甲酸的溶液条件下, 初 始浓度为 $5 \times 10^{-10} \mathrm{~mol} / \mathrm{L}$ 的 $\beta$-酪蛋白酶解液经材料富集 后仍可检出其中的一条多磷酸化肽(图5).

利用脱脂牛奶酶解液作为实际样品, 在添加有乙 酸的溶液条件下, 材料可以同时富集到包含单磷酸化 肽和多磷酸化肽在内的共计 13 条磷酸化肽(图6A); 而 在添加有甲酸的溶液中, 材料可以选择性地富集其中 的7条多磷酸化肽(图6B). 这一结果表明该材料在组成 较为复杂的实际样品中也有良好的应用效果, 可以根

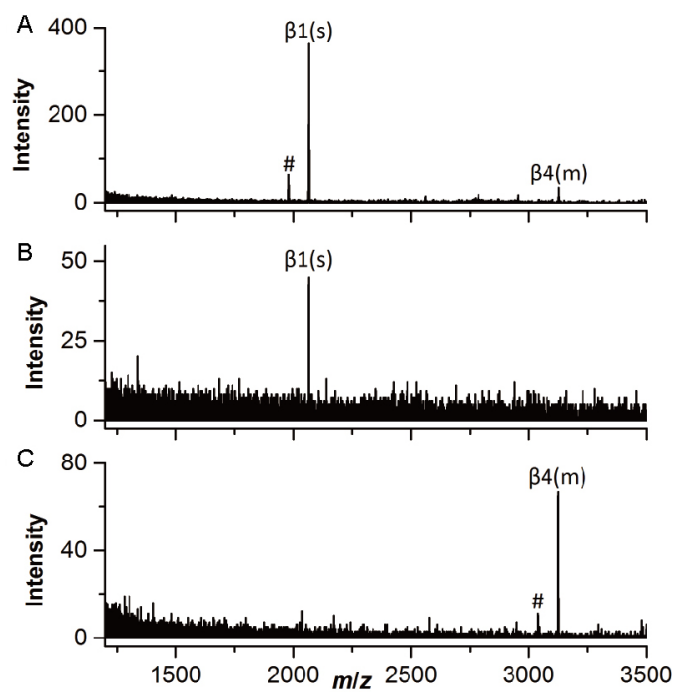

图 5 氨基功能化整体材料富集 $\beta$-酪蛋白酶解液中磷酸化 肽的检出限

上样溶液组成为和 $60 \%$ 乙腈(A)、 $0.05 \mathrm{~mol} / \mathrm{L}$ 乙酸(B)、 $60 \%$ 乙腈、 $1 \%$ 甲酸 $(\mathrm{C}) . \beta$ - 酪蛋白酶解液中的浓度为 $5 \times 10^{-10} \mathrm{~mol} / \mathrm{L}(\mathrm{A})$ 和 $(\mathrm{C})$; $2 \times 10^{-10} \mathrm{~mol} / \mathrm{L}(\mathrm{B})$

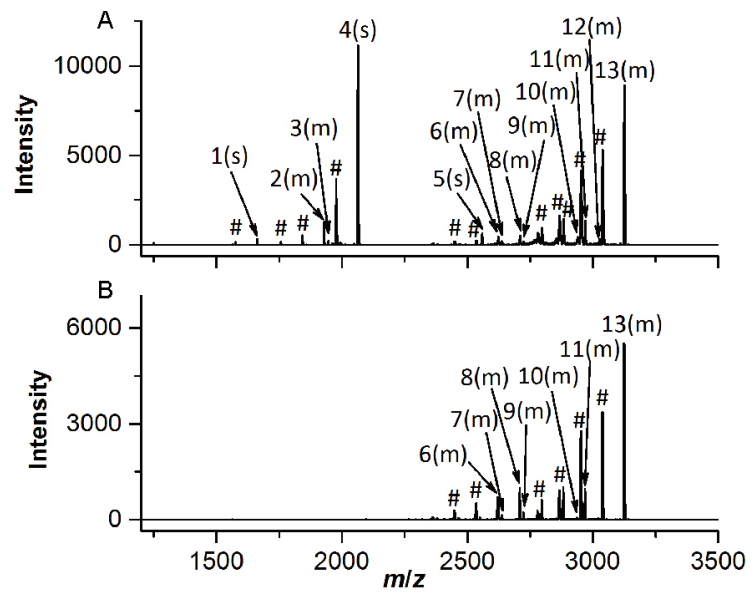

图 6 氨基功能化整体材料富集脱脂牛奶酶解液中磷酸化 肽的质谱图

$\mathrm{A}$ : 上样溶液组成为 $60 \%$ 乙腈、 $0.05 \mathrm{~mol} / \mathrm{L}$ 乙酸下的富集结果; B: 上 样溶液组成为 $60 \%$ 乙腈、 $0.5 \%$ 甲酸下的富集结果

据不同的富集需求实现对样品中磷酸化肽的全富集 或是对多磷酸化肽的选择性富集(表1).

\section{3 结论}

本研究利用原位聚合的方式, 在移液器吸头尖端 制备了氨基功能化整体材料, 并将这一材料应用于磷 
表 1 氨基功能化整体材料富集到的磷酸化肽信息 ${ }^{a)}$

\begin{tabular}{|c|c|c|c|}
\hline 编号 & 质荷比 ${ }^{*}$ & 磷酸化位点数目 & 序列 ${ }^{* *}$ \\
\hline$\beta 1$ & 2061.8 & 1 & FQpSEEQQQTEDELQDK \\
\hline$\beta 2$ & 2556.4 & 1 & FQpSEEQQQTEDELQDKIHPF \\
\hline$\beta 3$ & 2966.2 & 4 & ELEELNVPGEIVEpSLpSpSpSEESITR \\
\hline$\beta 4$ & 3122.3 & 4 & RELEELNVPGEIVEpSLpSpSpSEESITR \\
\hline 1 & 1660.8 & 1 & VPQLEIVPNpSAEER \\
\hline 2 & 1927.7 & 2 & DIGpSEpSTEDQAMEDIK \\
\hline 3 & 1943.7 & 2 & DIGpSEpSTEDQAoMEDIK \\
\hline 4 & 2061.8 & 1 & FQpSEEQQQTEDELQDK \\
\hline 5 & 2556.4 & 1 & FQpSEEQQQTEDELQDKIHPF \\
\hline 6 & 2618.9 & 4 & NTMEHVpSpSpSEEpSIISQETYK \\
\hline 7 & 2634.9 & 4 & NToMEHVpSpSpSEEpSIISQETYK \\
\hline 8 & 2704.9 & 5 & pyroQMEAEpSIpSpSpSEEIVPNpSVEQK \\
\hline 9 & 2720.9 & 5 & QMEAEpSIpSpSpSEEIVPNpSVEQK \\
\hline 10 & 2935.2 & 3 & EKVNELpSKDIGpSEpSTEDQAMEDIK \\
\hline 11 & 2966.2 & 4 & ELEELNVPGEIVEpSLpSpSpSEESITR \\
\hline 12 & 3025.5 & 2 & FPQpYLQpYLYQGPIVLNPWDQVKR \\
\hline 13 & 3122.3 & 4 & RELEELNVPGEIVEpSLpSpSpSEESITR \\
\hline
\end{tabular}

a) *: 质子化的单电荷准分子离子峰质荷比; **: 序列中“p”表示磷酸化氨基酸残基, “oM”表示氧化甲硫氨酸残基, “pyroQ”表示焦谷 氨酰胺残基

酸化肽富集. 该材料具有可控的磷酸化肽富集选择 性, 可在不同组成的上样溶液条件下分别实现对单磷 酸化肽和多磷酸化肽的同时富集以及对多磷酸化肽 的选择性富集, 因而是一种双功能磷酸化肽富集材料.
而借助于整体材料良好的通透性, 以及填充于吸头尖 端的应用形式, 磷酸化肽的富集操作得到了极大的简 化, 从而有效缩短了富集操作所需的时间, 在磷酸化 肽的快速富集分析中具有良好的应用前景.

\section{参考文献}

1 Van Hoof D, Muñoz J, Braam S R, et al. Phosphorylation dynamics during early differentiation of human embryonic stem cells. Cell Stem Cell, 2009, 5: 214-226

2 Duan D X, Chai G S, Ni Z F, et al. Phosphorylation of tau by death-associated protein kinase 1 antagonizes the kinase-induced cell apoptosis. J Alzheimers Dis, 2013, 37: 795-808

3 Augustinack J C, Schneider A, Mandelkow E M, et al. Specific tau phosphorylation sites correlate with severity of neuronal cytopathology in Alzheimer's disease. Acta Neuropathol, 2002, 103: 26-35

4 Wagner E F, Nebreda A R. Signal integration by JNK and p38 MAPK pathways in cancer development. Nat Rev Cancer, 2009, 9: 537-549

5 Mann M, Ong S E, Grønborg M, et al. Analysis of protein phosphorylation using mass spectrometry: deciphering the phosphoproteome. Trends Biotech, 2002, 20: 261-268

6 Boersema P J, Mohammed S, Heck A J. Phosphopeptide fragmentation and analysis by mass spectrometry. J Mass Spectrom, 2009, 44: 861-878

7 Hammarsten P, Karalija A, Josefsson A, et al. Low levels of phosphorylated epidermal growth factor receptor in nonmalignant and malignant prostate tissue predict favorable outcome in prostate cancer patients. Clin Cancer Res, 2010, 16: 1245-1255

8 Janek K, Wenschuh H, Bienert M, et al. Phosphopeptide analysis by positive and negative ion matrix-assisted laser desorption/ionization mass spectrometry. Rapid Commun Mass Spectrom, 2001, 15: 1593-1599 
9 Annesley T M. Ion suppression in mass spectrometry. Clin Chem, 2003, 49: 1041-1044

10 Zoumaro-Djayoon A D, Heck A J R, Muñoz J. Targeted analysis of tyrosine phosphorylation by immuno-affinity enrichment of tyrosine phosphorylated peptides prior to mass spectrometric analysis. Methods, 2012, 56: 268-274

11 Bian Y, Li L, Dong M, et al. Ultra-deep tyrosine phosphoproteomics enabled by a phosphotyrosine superbinder. Nat Chem Biol, 2016, 12: 959-966

12 Helling S, Shinde S, Brosseron F, et al. Ultratrace enrichment of tyrosine phosphorylated peptides on an imprinted polymer. Anal Chem, 2011, 83: $1862-1865$

13 Dong M, Wu M, Wang F, et al. Coupling strong anion-exchange monolithic capillary with MALDI-TOF MS for sensitive detection of phosphopeptides in protein digest. Anal Chem, 2010, 82: 2907-2915

14 Zhou H J, Ye M L, Dong J, et al. Robust phosphoproteome enrichment using monodisperse microsphere-based immobilized titanium (IV) ion affinity chromatography. Nat Protoc, 2013, 8: 461-480

15 Larsen M R, Thingholm T E, Jensen O N, et al. Highly selective enrichment of phosphorylated peptides from peptide mixtures using titanium dioxide microcolumns. Mol Cell Proteomics, 2005, 4: 873-886

16 Messner C B, Mirza M R, Rainer M, et al. Selective enrichment of phosphopeptides by a metal-organic framework. Anal Methods, 2013, 5: 2379-2383

17 Qiao L, Bi H Y, Busnel J M, et al. Controlling the specific enrichment of multi-phosphorylated peptides on oxide materials: aluminium foil as a target plate for laser desorption ionization mass spectrometry. Chem Sci, 2010, 1: 374-382

18 Fleitz A, Nieves E, Madrid-Aliste C, et al. Enhanced detection of multiply phosphorylated peptides and identification of their sites of modification. Anal Chem, 2013, 85: 8566-8576

19 Li L P, Zheng T, Xu L N, et al. $\mathrm{SnO}_{2}-\mathrm{ZnSn}(\mathrm{OH})_{6}$ : a novel binary affinity probe for global phosphopeptide detection. Chem Commun, 2013, 49: 1762-1764

20 Shiau K J, Hung S U, Lee H W, et al. Nanodiamond-based two-step sampling of multiply and singly phosphorylated peptides for MALDI-TOF mass spectrometry analysis. Analyst, 2011, 136: 1922-1927

21 Xu L N, Li L P, Jin L, et al. Guanidyl-functionalized graphene as a bifunctional adsorbent for selective enrichment of phosphopeptides. Chem Commun, 2014, 50: 10963-10966

22 Xu L, Ma W, Shen S, et al. Hydrazide functionalized monodispersed silica microspheres: a novel probe with tunable selectivity for a versatile enrichment of phosphopeptides with different numbers of phosphorylation sites in MS analysis. Chem Commun, 2016, 52: 1162-1165

23 Shi C, Deng C. Immobilized metal ion affinity chromatography ZipTip pipette tip with polydopamine modification and $\mathrm{Ti}^{4+}$ immobilization for selective enrichment and isolation of phosphopeptides. Talanta, 2015, 143: 464-468

$24 \mathrm{Hu}$ Y, Shan C X, Wang J, et al. Fabrication of functionalized $\mathrm{SiO}_{2} / \mathrm{TiO}_{2}$ nanocomposites via amidation for the fast and selective enrichment of phosphopeptides. New J Chem, 2015, 39: 6540-6547

25 Guiochon G. Monolithic columns in high-performance liquid chromatography. J Chromatogr A, 2007, 1168: 101-168

26 Yu C, Davey M H, Svec F, et al. Monolithic porous polymer for on-chip solid-phase extraction and preconcentration prepared by photoinitiated in situ polymerization within a microfluidic device. Anal Chem, 2001, 73: 5088-5096 


\title{
Amino-functionalized monolithic tips for rapid phosphopeptide enrichment with tunable selectivity
}

\author{
XU LinNan ${ }^{1,2}$, BAI Yu ${ }^{1} \&$ LIU HuWei ${ }^{1}$ \\ 1 Beijing National Laboratory for Molecular Sciences, Key Laboratory of Bioorganic Chemistry and Molecular Engineering of Ministry of \\ Education, Institute of Analytical Chemistry, College of Chemistry and Molecular Engineering, Peking University, Beijing 100871, China; \\ 2 Beijing Institute of Aerospace Testing Technology, Beijing 100074, China
}

To overcome the concern pertaining to low sensitivity in phosphopeptide analysis by mass spectrometry, amino-functionalized monolithic tips were designed for rapid phosphopeptide enrichment. Phosphopeptide enrichment using amino-functionalized monolithic tips featured ease of operation and low time consumption and helped preventing sample loss caused by incomplete separation of material and solution. In solutions with different compositions, the synthesized monolithic tips showed different enrichment selectivity toward mono- and multiphosphopeptides. Both global enrichment of all phosphopeptides and selective enrichment of multiphosphopeptides were realized by modulating the composition of solution for sample loading.

amino-functionalized monolithic tip, phosphopeptide, rapid enrichment, tunable selectivity

doi: $10.1360 / \mathrm{N} 052017-00171$ 